\title{
Extending two-dimensional histology into the third dimension through conventional micro computed tomography
}

Anna Khimchenko ${ }^{a}$, Hans Deyhle ${ }^{\mathrm{a}}$, Georg Schulz ${ }^{\mathrm{a}}$, Gabriel Schweighauser ${ }^{\mathrm{c}}$, Jürgen Hench ${ }^{\mathrm{c}}$, Natalia Chicherova $^{\mathrm{a}, \mathrm{b}}$, Christos Bikis ${ }^{\mathrm{a}}$, Simone E. Hieber ${ }^{\mathrm{a}}$, Bert Müller ${ }^{\mathrm{a}, *}$

${ }^{a}$ Biomaterials Science Center, Department of Biomedical Engineering, University of Basel, Allschwil, Switzerland

${ }^{b}$ Medical Image Analysis Center, Department of Biomedical Engineering, University of Basel, Allschwil, Switzerland ${ }^{c}$ Institute of Pathology, Department of Neuropathology, Basel University Hospital, Basel, Switzerland

\begin{abstract}
Histological examination achieves sub-micrometer resolution laterally. In the third dimension, however, resolution is limited to section thickness. In addition, histological sectioning and mounting sections on glass slides introduce tissue-dependent stress and strain. In contrast, state-of-the-art hard X-ray microcomputed tomography $(\mu \mathrm{CT})$ systems provide isotropic sub-micrometer resolution and avoid sectioning artefacts. The drawback of $\mu \mathrm{CT}$ in the absorption contrast mode for visualising physically soft tissue is a low attenuation difference between anatomical features. In this communication, we demonstrate that formalin-fixed paraffin-embedded human cerebellum yields appropriate absorption contrast in laboratorybased $\mu \mathrm{CT}$ data, comparable to conventional histological sections. Purkinje cells, for example, are readily visible. In order to investigate the pros and cons of complementary approaches, two- and three-dimensional data were manually and automatically registered. The joint histogram of histology and the related $\mu \mathrm{CT}$ slice allows for a detailed discussion on how to integrate two-dimensional information from histology into a threedimensional tomography dataset. This methodology is not only rewarding for the analysis of the human cerebellum, but it also has relevance for investigations of tissue biopsies and post-mortem applications. Our data indicate that laboratory-based $\mu \mathrm{CT}$ as a modality can fill the gap between synchrotron radiationbased $\mu \mathrm{CT}$ and histology for a variety of tissues. As the information from haematoxylin and eosin (H\&E) stained sections and $\mu \mathrm{CT}$ data is related, one can colourise local X-ray absorption values according to the $\mathrm{H} \& \mathrm{E}$ stain. Hence, $\mu \mathrm{CT}$ data can correlate and virtually extend two-dimensional (2D) histology data into
\end{abstract}


the third dimension.

Keywords: Hard X-ray tomography, nano-focus X-ray, histology, 2D-3D image registration, paraffin-embedded human cerebellum, Purkinje cells, joint histogram analysis.

\section{Introduction}

The microanatomy, i.e. microstructures and morphology, of tissue components is generally characterised by means of histological sectioning, as this sort of examination can provide a true (sub-)micrometer resolution in two lateral dimensions, when neglecting processing-derived shrinkage artefacts, and the option of functional staining for the contrast (Müller et al., 2006, Irshad et al., 2014). By means of optical microscopy individual intra- and extracellular components are visualised (Irshad et al., 2014, Kandel et al. 2012. Fuchs and Buhmann, 2011). Currently established protocols, however, are often time-consuming, and individual steps involved in the preparation procedure induce stress- and strain-related artefacts in the tissue (Schulz et al. 2010a; Müller et al. 2012, Germann et al., 2008). Moreover, for histological examinations, the tissue has to be irreversibly cut into slices (Lang et al., 2014). As the sections are a few micrometres thick, isotropic lateral resolution is lost in the third dimension. Therefore, non-destructive three-dimensional imaging is a promising complement to providing volumetric morphological information (Schulz et al. 2010a).

Confocal microscopy yields images of cells in layers well below the surface. However, the limited transmission of visible light does not allow for the comprehensive visualisation of human tissue in its threedimensional (3D) state (Müller et al., 2006). Multiphoton fluorescence in general and one- or two-photon microscopy (Wolf et al., 2015) in particular can provide information from tissue layers up to hundreds of micrometers, while decreasing the spatial resolution (So, 2002), for example zebrafish larval brain (Vladimirov et al., 2014). Tissue-clearing methods significantly increase the accessible depth (Richardson and Lichtman, 2015). For example, tissue-transformation method CLARITY can increase the achievable imaging depth up to 5 or even $6 \mathrm{~mm}$ (Chung et al., 2013). The SWITCH method improves the light penetration as well, demonstrating clearing of a whole adult mouse brain, lung, kidney, heart, liver and spinal cord with a required tissue-clearing time between five and ten days (Murray et al. 2015). Combining tissue clearing with confocal light sheet and light sheet fluorescence microscopies allows for the visualization of entire mouse brains (Dodt et al. 2007; Silvestri et al., 2012; Costantini et al., 2015). Nevertheless, these proce- 
dures are often technically demanding, expensive, time-consuming, induce significant tissue deformation, and restricted to a particular tissue type with sizes not exceeding a thickness of some millimetres.

Another means of obtaining 3D morphology is serial sectioning, which is time-consuming and is mainly applied to small tissue volumes (Chung et al., 2013). The time restriction can be overcome by applying a serial optical coherence scanner (Wang et al., 2014), albeit spatial resolution in the third dimension still remains restricted to section thickness (Lang et al., 2014; Schulz et al. 2010a). Nonetheless, these methods are destructive and the same sample can often not be reused for subsequent examinations.

Synchrotron radiation-based micro and nano computed tomography ( $\mathrm{SR} \mu \mathrm{CT}$ ) provides impressive 3D images of biological tissues on a (sub-)cellular level (Zehbe et al., 2010; Huang et al., 2015). For example, one can detect and image RNA/DNA-stained HEK 293 cell clusters (Müller et al., 2006), intracellular structures of dehydrated human cells (Guk et al., 2008), single endothelial cells labelled with iron oxide particles (Thimm et al., 2012), chondrocytes within the extracellular matrix of articular cartilages without metal staining (Zehbe et al. 2015), the lacuno-canalicular network and collagen fibres in human bone (Langer et al., 2012), osmium-stained individual ganglion cells (Lareida et al., 2009), unstained Purkinje cells (Schulz et al. 2010b) and freeze-dried neurons (Mokso et al., 2007). The limited accessibility of synchrotron radiation facilities, though, imposes severe restrictions on the user (Wenz et al., 2015).

In contrast to laboratory sources, the synchrotron radiation sources yield such a high photon flux, that a monochromator can be incorporated to pass about $10^{-4}$ of the photons and generate monochromatic light, avoiding beam hardening. Laboratory-based $\mu \mathrm{CT}$ systems have successfully been employed for 3D visualisation of higher density materials (Chappard et al., 2005, Blouin et al., 2006) and stained tissues (Metscher, 2009; de Crespigny et al., 2008; Ribi et al., 2008; Ashton et al., 2015). Recently $\mu \mathrm{CT}$ was successfully used for analysis of brain architecture of insect species (Sombke et al., 2015) . Furthermore, $\mu \mathrm{CT}$ can achieve superb resolution, although performance for lower density materials is restricted due to limited contrast.

Consequently, we state that there is a paucity of methods to study the microstructure and morphology of large tissue components in 3D space with isotropic (sub-)cellular spatial resolution within a laboratory environment.

In this study, we evaluate the contrast of formalin-fixed paraffin-embedded (FFPE) tissue obtained with laboratory-based $\mu \mathrm{CT}$. We aim to demonstrate the three-dimensional non-destructive visualisation 
of a human cerebellum sample with cellular resolution, with phoenix $\mid$ xray nanotom ${ }^{\circledR}$ m laboratory-based $\mu \mathrm{CT}$ system.

In order to directly compare three-dimensional $\mu \mathrm{CT}$ data with histology, the counterpart of the histological section has to be localised within the three-dimensional $\mu$ CT dataset (Stalder et al., 2014). Such registration enables the validation of structures within the $\mu \mathrm{CT}$ data by selected histological sections (Gambichler et al., 2007).

It was shown that synchrotron radiation-based computed tomography, using the phase-contrast mode, allows for identifying not only major blood vessels, but also Stratum moleculare, Stratum granulosum and white matter within formalin-fixed human cerebellum - even individual Purkinje cells are visualised (Schulz et al. 2010b). The question arises as to whether laboratory-based absorption-contrast $\mu \mathrm{CT}$ of an FFPE human cerebellum sample can provide comparable results. We investigate how $\mu \mathrm{CT}$ could become a complementary method to the microscopic examination of stained tissue slices, thereby extending its applicability to three-dimensional features.

\section{Materials and methods}

\subsection{Tissue preparation}

The specimen was extracted from the donated cadaveric brain of a 73-year-old man. Written consent for scientific use was documented. All procedures were conducted in accordance with the Declaration of Helsinki and approved by the Ethikkommission Nordwestschweiz. The brain was fixed in $4 \%$ histologicalgrade buffered formalin for two weeks prior to dissection. Tissue samples for histological work-up were excised by a scalpel. These specimens, approximately $15 \mathrm{~mm} \times 15 \mathrm{~mm} \times 4 \mathrm{~mm}$, to fit into conventional histological embedding cassettes, were dehydrated and paraffin embedded according to surgical pathology procedures: $3 \times 1$ hour in $70 \%$ ethanol $(\mathrm{EtOH})$ in $\mathrm{H}_{2} \mathrm{O}(\mathrm{v} / \mathrm{v}), 1$ hour in $80 \%$ EtOH in $\mathrm{H}_{2} \mathrm{O}(\mathrm{v} / \mathrm{v}), 2 \times 1$ hour in $96 \%$ EtOH in $\mathrm{H}_{2} \mathrm{O}(\mathrm{v} / \mathrm{v}), 2 \times 1$ hour $100 \%$ xylene followed by $3 \times 1$ hour paraffin/plastic mixture (Surgipath Paraplast ${ }^{\circledR}$, Leica Biosystems, Switzerland) at $60^{\circ} \mathrm{C}$. Next, samples within the molten paraffin were transferred to histological embedding moulds on a routine paraffin block-casting device. Here, the paraffin was cooled down to approximately $-8^{\circ} \mathrm{C}$, to solidify and then removed from the casting moulds. Cylinders $6 \mathrm{~mm}$ in diameter were extracted from the FFPE tissue, using a metal punch, for $\mu \mathrm{CT}$ measurements. 
82

Typically, minimum formaldehyde fixation time is one hour, paraffin embedding takes approximately ten hours, casting blocks five minutes and punching five minutes.

\subsection{Data acquisition and processing}

The tomography experiments were carried out using the absorption-contrast $\mu \mathrm{CT}$ system nanotom ${ }^{\circledR} \mathrm{m}$ (phoenix|x-ray, GE Sensing \& Inspection Technologies GmbH, Wunstorf, Germany) equipped with a $180 \mathrm{kV}$ ¿ $15 \mathrm{~W}$ high-power nano-focus ${ }^{\circledR}$ tube with W and Mo transmission targets General Electric, Measurement and Control, 2014; Egbert and Brunke, 2011). The nanotom ${ }^{\circledR} \mathrm{m} \mu \mathrm{CT}$-system allows measuring objects with up to $25 \mathrm{~cm}$ in diameter and height.

The $\mu \mathrm{CT}$ was performed with a voxel length of $3.5 \mu^{3}$ and a field of view of about $8.5 \times 10.5 \mathrm{~mm}^{2}$. For each acquisition, 1900 projections were recorded over $360^{\circ}$. Geometric magnification $M$ was maintained at 28.57 for selected focus-detector distances (FDD) and focus-object distances (FOD): $M=$ FDD $/ F O D$. Measurements were taken in the tube operation mode " 0 " with an estimated source size of 2.7 um.

Data acquisition and reconstruction were performed with datos $\mid \mathrm{x} 2.0$ software (phoenix|x-ray, GE Sensing \& Inspection Technologies GmbH, Wunstorf, Germany). The average data acquisition time was 3.5 hours, with the requirement of the user interaction in the beginning of the scan followed by the automated execution. Data reconstruction is based on Feldkamps cone beam reconstruction algorithm $($ Egbert and Brunke, 2011; Feldkamp et al., 1984) and can be automatically done within less then 15 minutes. The reconstructed slices were scaled from black to white within the range of three times the distance from the maximum to the minimum histogram peak position for each dataset.

The reconstructed datasets were compared for their contrast-to-noise ratio:

$$
C N R=\frac{\left|I_{1}-I_{2}\right|}{\sqrt{\sigma_{1}^{2}+\sigma_{2}^{2}}}
$$

where $I_{1}$ and $I_{2}$ indicate the mean intensities of homogeneous components within the specimen, and $\sigma_{1}$ and $\sigma_{2}$ the standard deviations. To this end, volumes of interest (VOI) were selected within the white matter (VOI=18200 voxels), Stratum granulosum (VOI=5292 voxels), Stratum moleculare (VOI=4032 voxels), and paraffin $(\mathrm{VOI}=4704$ voxels) of each dataset. To ensure comparability of the $C N R$ values, VOIs were selected from the same location within each dataset. 
Reconstructed data were filtered using VGStudio MAX 2.0 (Volume Graphics, Heidelberg, Germany) for noise reduction (median filter size " 5 " followed by an adaptive Gauss filter with smoothing "0.1" and an edge threshold "1") for comparison with histology.

\subsection{Histology}

Subsequent to the laboratory-based $\mu \mathrm{CT}$ measurements, the specimen was investigated by means of histology. The paraffin cylinder was re-embedded in a standard histological paraffin block by melting it, placing the tissue cylinder into a mound and filling it up with fresh paraffin. Sections of $4 \mu \mathrm{m}$ thickness were cut using a microtome, mounted on glass slides, and stained with haematoxylin and eosin (H\&E). The preparation time ranged from 30 to $60 \mathrm{~s}$ per slide.

Images of the resulting slides were taken on a light microscope and resulted in pixel sizes of $6.99 \mu \mathrm{m}$ and $4.38 \mu \mathrm{m}$. These pixel sizes were selected to become comparable to the $\mu \mathrm{CT}$ data.The resolution of computed tomography depends on several factors including the source spot size due to focal spot blurring effect, noise on a detector and beam instability and is normally within the range of 1.5 to 2.0 pixel sizes. Under such estimation, resolutions of the selected histological section and tomography slice are comparable. Micrographs acquired with the slide scanner (Olympus ${ }^{\circledR}$ VS120 Virtual Slide Microscope, Japan) reached $0.35 \mu \mathrm{m}$ pixel size.

A histological photograph with a pixel size of $6.99 \mu \mathrm{m}$ was used for the quantitative comparison to the tomography slice. For comparison, the histological section was grey-scaled and colour-inverted to provide grey-value correlation to the tomography data.

\subsection{Data registration}

To locate the histological slide within the $\mu \mathrm{CT}$ data, 2D-3D registration was performed Chicherova et al. 2014). First, corresponding feature points between the histological image and each image in the $\mu \mathrm{CT}$ volume were identified using the key-point detector SURF (Bay et al., 2008). The coordinates of the matching points of the $\mu \mathrm{CT}$ images were stored in a 3D space. Second, the points were filtered according to their density. Weights to each of the points in the 3D cloud were assigned based on their neighborhood. The 500 points with the highest weights were selected. Finally, a plane was fitted into the filtered 3D cloud using the modified model-fitting algorithm RANSAC (Fischler and Bolles, 1981). The corresponding tomogram is then interpolated from the $\mu \mathrm{CT}$ volume using the obtained coordinates of the 
plane, see Fig. 1. After matching the histological slide in the tomogram, we registered the histology section and correlated tomography slice in 2D, using the Demon registration tool (Kroon and Slump, 2009) with affine transformation constraint. In the present study, the histological slides show only gradually varying local deformations. Therefore, one may conclude that the affine transformation is sufficiently precise to accurately register histology slide with $\mu \mathrm{CT}$ slice. We took the histological section as the reference, due to its higher spatial resolution, and the tomographic slice as the floating image. The entire pipeline was written in Matlab R2014a (MathWorks, Natick, USA).

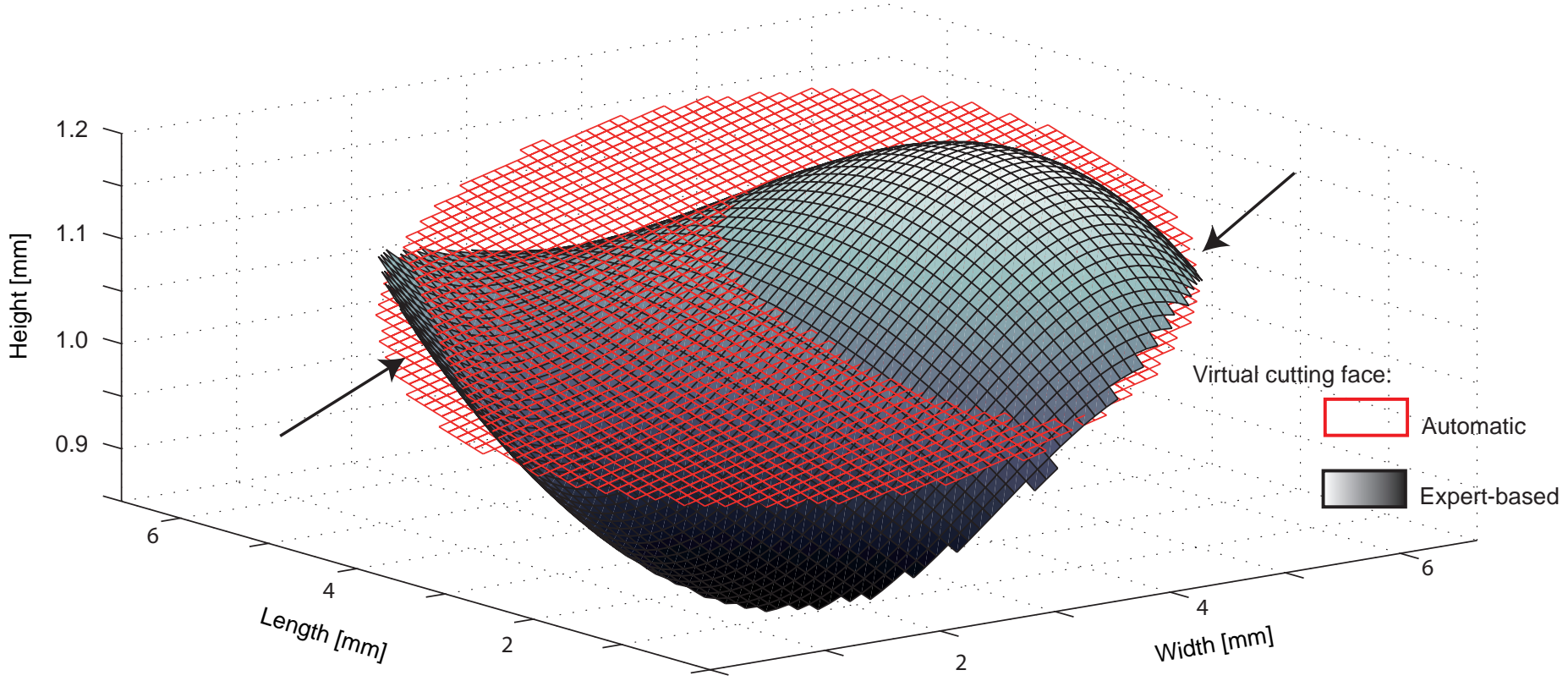

Fig. 1: Shape of the histological section in the tomography dataset based on the expert-based (black) and automatic (red) registrations. Note that automatic registration yields a plane, while manual registration allows for curved surfaces. Arrows indicate regions of low landmark density.

To validate automatic registration, an expert-based 2D-3D registration was performed. The registration was based on the point-to-point correspondence of anatomical landmarks located in tomography data and histology images (Markelj et al. 2012) (see Fig. 2). For this purpose, characteristic vessels, cell groups and cracks seen in both datasets were matched in four histological sections. The manually identified landmarks in the tomography data were then fitted with a 2D polynomial surface (see Fig. 11), using the Curve Fitting Toolbox $^{T M}$ implemented in Matlab R2014a. This surface was considered to correspond to the location of the histological section in the 3D data. 2D-2D affine post-registration of the histological section and its 
tomographic counterpart was performed in a manner similar to that used for automatic registration.
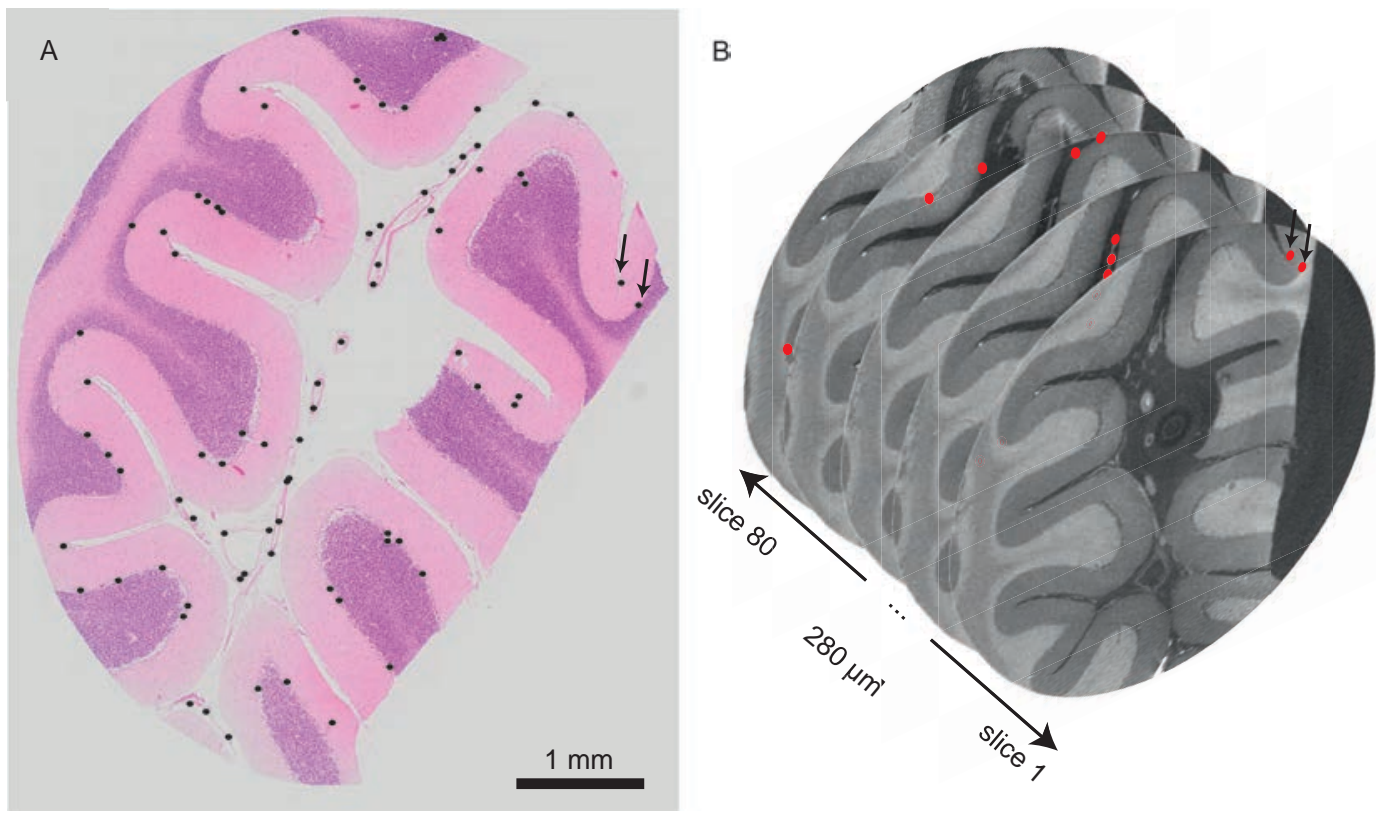

Fig. 2: Visualisation of characteristic landmarks for manual 2D-3D registration marked in a selected histological section (A) and in the $\mu \mathrm{CT}$ dataset (B). Characteristic landmarks of one histological section are spread over 80 consecutive $\mu \mathrm{CT}$ slices.

\subsection{Volume ratio calculation}

The volume ratio (VRs) of structures of interest was calculated, defined as: $V R=V_{1} / V_{2}$, where $V_{1}$ and $V_{2}$ indicate the volume/area occupied by structures of interest within the sample in voxels/pixels. We calculated the surface area (in pixels) occupied by Stratum moleculare/white matter and Stratum granulosum for the registered tomographic volume $(*)$, and the selected histological section and tomographic slice. The surface area was extracted from histogram based segmentation (Manjon-Herrera, 2006). In order to calculate the volume ratio for 3D data, cumulative slice-wise calculation was carried out.

\subsection{Histogram analysis}

The quantitative comparison of histology and tomography was based on the histogram analysis of the selected registered slices. For the quantitative analysis of the registered $\mu \mathrm{CT}$ and histology slices, the grey value histograms of the corresponding 2D images were fitted with multi-Gaussian distributions (Müller et al., 2002), using the Levenberg-Marquardt algorithm of OriginPro 7.5 (OriginLab Corporation, Northampton, USA) (Schulz et al. 2010b, 2012). This common approach supports the intensity-based 
163

\subsection{Parameter selection for optimised tomography}
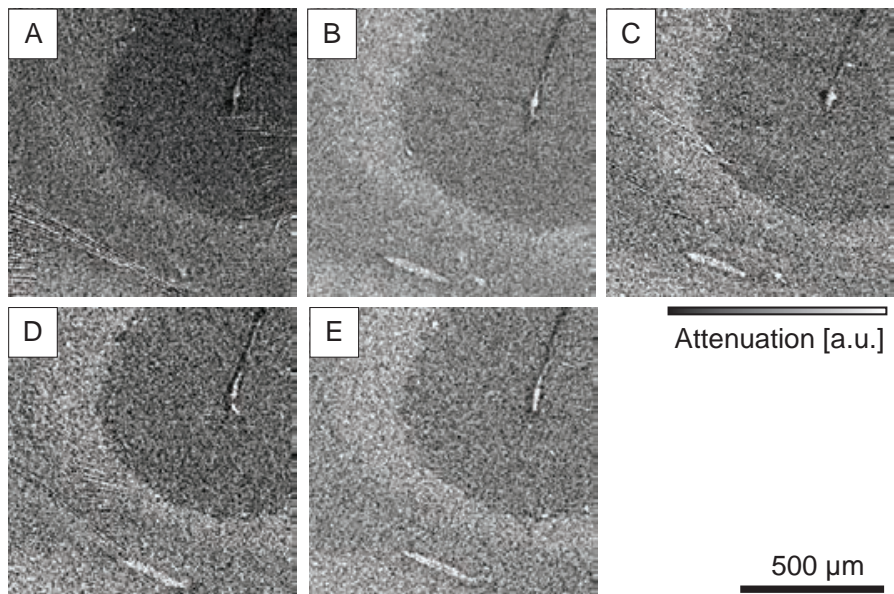

Attenuation [a.u.]
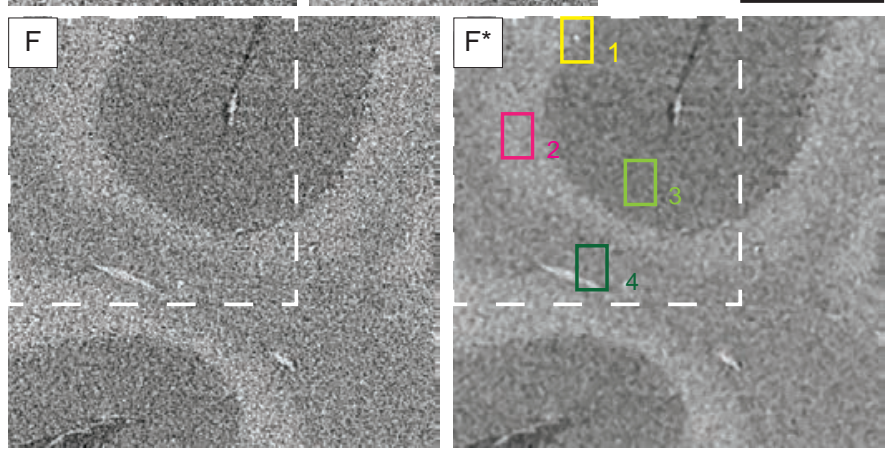

Fig. 3: Human cerebellum block measured with six $\mu \mathrm{CT}$ settings. Images provide sufficient contrast for visualising morphological features. As the images are dominated by noise, filtering is required. A-F: selected part of the tomographic slice recorded with parameters summarised in Table 1. F*: Slice F filtered for noise reduction. 1 (yellow): Purkinje cell, 2 (pink): Stratum granulosum, 3 (light green): Stratum moleculare, 4 (dark green): blood vessel within the white matter.

Fig. 3 presents parts of $\mu \mathrm{CT}$ slices acquired with the parameters summarised in Table 1 . The slices in Fig. 3 are cropped around the region occupied by three structures of interest. Visual inspection reveals that Stratum granulosum (2, pink), Stratum moleculare (3, light green), individual cells (1, yellow), and blood vessels within the white matter (4, dark green) can be recognised. Images obtained with increased FDD have significant artefacts, resulting in a radial grey-value gradient. Since the $\mu \mathrm{CT}$ raw data comprises 
noise, filtering is essential for feature extraction. The impact of filtering can for example be recognized comparing the images in Fig. $3 \mathrm{~F}$ and Fig. $3 \mathrm{~F}^{*}$.

The reconstructed datasets, recorded with the parameters summarised in Table 1, were compared for their contrast-to-noise ratio. The highest $C N R$ for the selected anatomical features was achieved for scans $\mathrm{B}$ and F, and differences between the scans are within the confidence interval. Even though the $C N R$ for scan B is higher than for scan F by trend, data from scan F was filtered and used for further analysis, since the acquisition time was approximately 2.5 hours and was half that of scan B.

\section{Table 1}

Selection of the optimized settings: Scanning parameters of the nanotom ${ }^{\circledR} \mathrm{m}$ used for the $\mu \mathrm{CT}$ experiments and contrast-tonoise ratios (CNRs) of the reconstructed data. Target: X-ray tube transmission target, $U$ : acceleration voltage, $I$ : e-beam current, FDD: focus-detector distance, FOD: focus-object distance, $t$ : exposure time per projection, CNR $(I)$ : contrast-tonoise ratio between paraffin and Stratum moleculare; CNR $(I I)$ : contrast-to-noise ratio between paraffin and white matter; CNR(III): contrast-to-noise ratio between paraffin and Stratum granulosum.

\begin{tabular}{cccccccccc}
\hline Setting & Target & $\boldsymbol{U}$ & $\boldsymbol{I}$ & FDD & FOD & $\boldsymbol{t}$ & CNR & $\begin{array}{c}\text { CNR } \\
{[I I)}\end{array}$ & $\begin{array}{c}\text { CNR } \\
(I I I)\end{array}$ \\
\hline $\mathrm{A}$ & $\mathrm{Mo}$ & 40 & 230 & 285.69 & 9.99 & 7 & $0.02 \pm 0.07$ & $1.26 \pm 0.07$ & $1.42 \pm 0.07$ \\
$\mathrm{~B}$ & $\mathrm{~W}$ & 40 & 350 & 285.69 & 9.99 & 6 & $0.88 \pm 0.20$ & $1.35 \pm 0.21$ & $1.91 \pm 0.23$ \\
$\mathrm{C}$ & $\mathrm{W}$ & 40 & 350 & 399.99 & 13.99 & 12 & $0.44 \pm 0.09$ & $0.87 \pm 0.09$ & $1.28 \pm 0.09$ \\
$\mathrm{D}$ & $\mathrm{Mo}$ & 50 & 180 & 285.69 & 9.99 & 7 & $0.31 \pm 0.07$ & $0.70 \pm 0.07$ & $1.11 \pm 0.07$ \\
$\mathrm{E}$ & $\mathrm{Mo}$ & 60 & 150 & 285.69 & 9.99 & 3 & $0.68 \pm 0.06$ & $1.01 \pm 0.06$ & $1.45 \pm 0.07$ \\
$\mathrm{~F}$ & $\mathrm{~W}$ & 60 & 350 & 285.69 & 9.99 & 3 & $0.70 \pm 0.10$ & $1.11 \pm 0.17$ & $1.57 \pm 0.18$ \\
\hline
\end{tabular}

The 3D-rendering of filtered $\mu \mathrm{CT}$ data in Fig. 4 shows the 3D micro-architecture of the selected part of the FFPE human cerebellum. It is illustrated that the morphology of the human tissue sample can be investigated using laboratory-based $\mu \mathrm{CT}$ on a cellular level, without applying contrast agents. After filtering and intensity thresholding, segmentation allows for precise discrimination of Stratum moleculare and Stratum granulosum. The diameters of the vessel in Fig. $4 \mathrm{C}$ and Purkinje cell in Fig. 4 D were estimated based on the number of voxels in the related orthogonal cutting plane manually. 


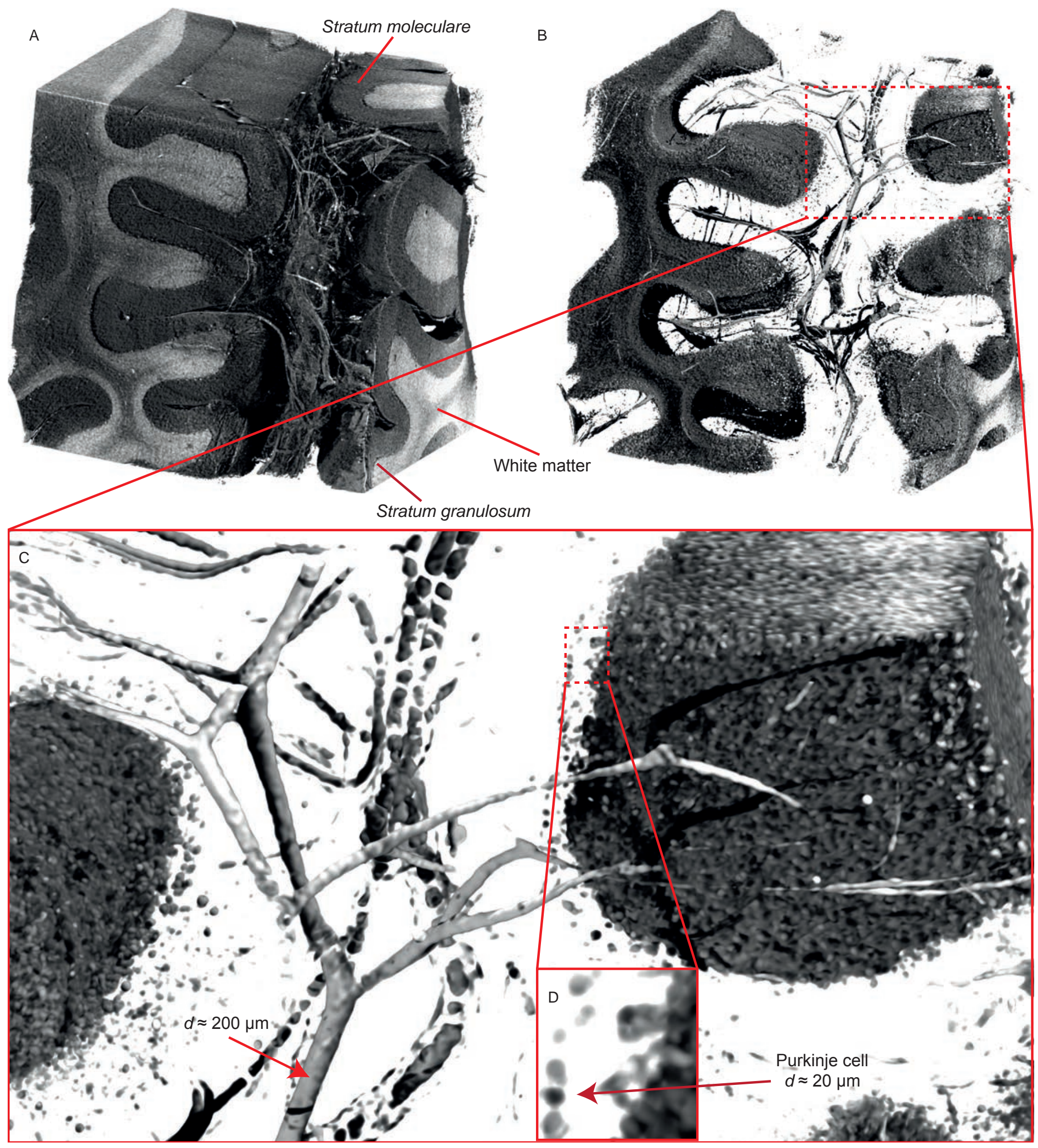

Fig. 4: The 3D-rendering of the human cerebellum block based on the filtered $\mu \mathrm{CT}$ data $(\mathrm{A})$ shows blood vessels of various sizes, Stratum moleculare, Stratum granulosum, and white matter. Intensity thresholding (B) enables the exclusion of Stratum moleculare for an improved visualisation of the blood vessels and Purkinje cells (C, D). 


\subsection{Retrieval of histology slice in $\mu C T$ data}

An established technique to investigate anatomical structures is histology. Based on size, location, and shape one could reasonably assume that the elliptically shaped features in the $\mathrm{\mu CT}$ data correspond to the Purkinje cells. The four histology slides validated this assumption in 2D.
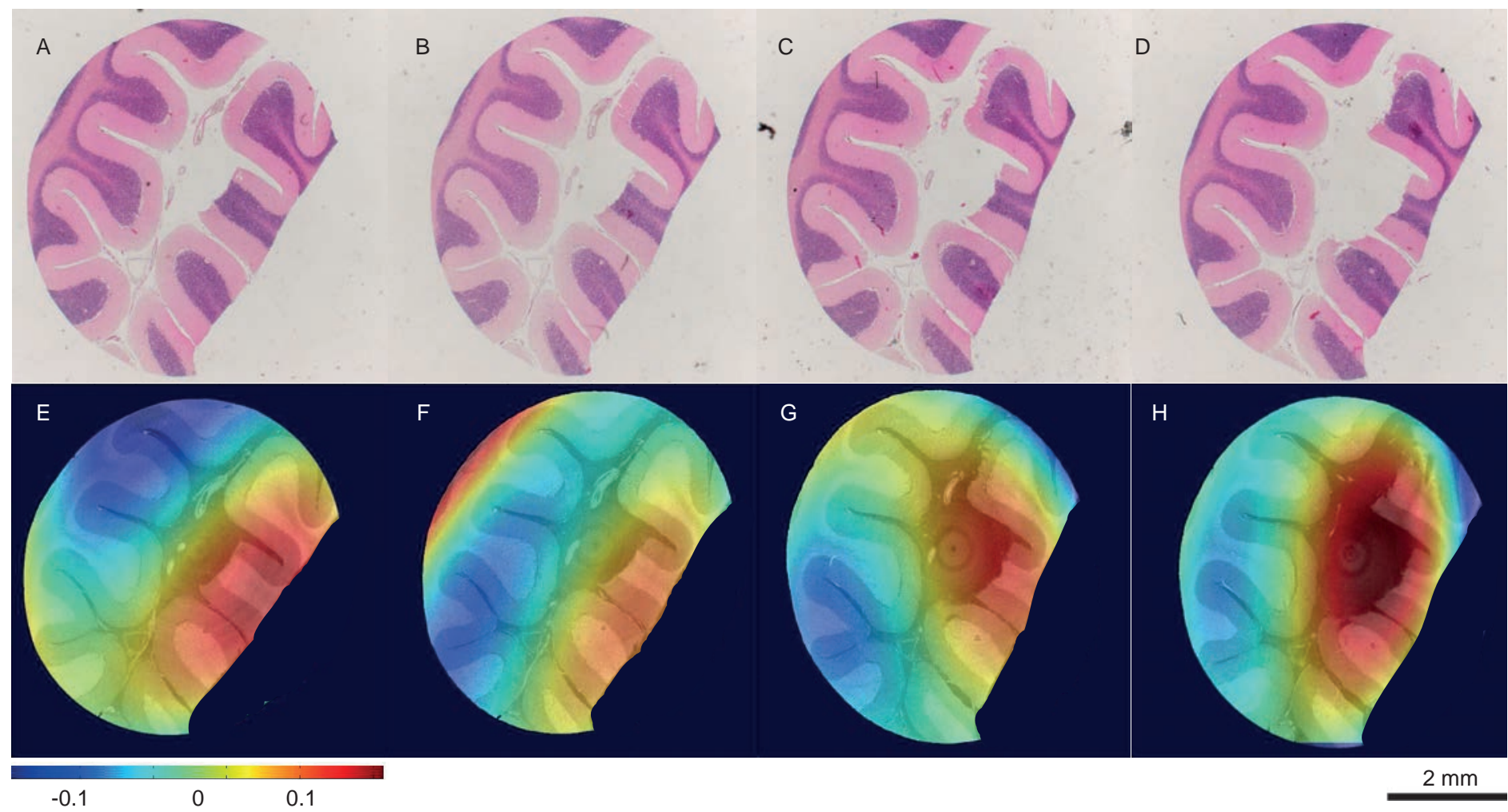

Height [mm]

Fig. 5: Virtual cutting planes within the $\mu \mathrm{CT}$ volume visualising the position of the histological sections. The colour on top of the registered $\mu \mathrm{CT}$ slices $(\mathrm{E}-\mathrm{H})$ indicates the shape of the histological sections $(\mathrm{A}-\mathrm{D})$ in the tomography dataset based on the expert-based registration.

The polynomial surface fit provides reasonable results in regions of high landmark point density; however, it diverges quickly outside these regions. Regions presenting a limited number, or absence of, landmarks are comparatively small. Thus, these differences are not pronounced, and the visual quality of the registration is preserved. For example, in regions labelled with an arrow in Fig. 1, the surface has a steep slope, and the fitting quality deteriorates. However, this deviation is limited to the homogeneous border regions of the slice. The slopes of our virtual histology sections are more prominent in regions of low landmark density, e.g. in a homogeneous middle background part that does not have any features in the 
histological section (see Fig. 5, G and H central parts).

Manual 2D-3D registration of a single histological slide to $\mu \mathrm{CT}$ volume is highly time consuming process that lasts up to 8 hours for identification of point-to-point correspondence of anatomical landmarks. Thus, manual registration was done only for validation purposes. Precision of the automatic 2D-3D registration can be also deduced from Fig. 6, if one considers the expert-based registration as ground truth. Mean difference between manual and automatic registrations planes is $4 \mu \mathrm{m}$.

The characteristic landmarks used for the expert-based 2D-3D registration are distributed over 80 tomographic slices (see Fig. 2, which results in a height difference of 280 um over a $6 \mathrm{~mm}$ sample diameter. Thus, the tilting angle corresponds to $2.6^{\circ}$. The tilting angle for the virtual cutting plane based on automatic registration is around $3^{\circ}$, while the maximal tilting angle for the histological cut is $5^{\circ}$ and estimated at around $1^{\circ}$ to $3^{\circ}$, therefore being in agreement with our findings.

To evaluate distortions in the selected histological slide, an additional 2D-2D registration with the tomographic slice as the reference and the histological section as the floating image was performed. A comparison of uncorrected and corrected histological sections enables one to conclude that radial stretching by approximately $6 \%$ and longitudinal stretching by approximately $15 \%$ have taken place.

\subsection{Comparison of tomography and light microscopy-based imaging of histological sections}

Fig. 6 displays the human cerebellum, with manually (left) and automatically (right) registered tomography slices compared to the selected high resolution histological section with a pixel size of $0.34 \mu \mathrm{m}$ (middle) at three magnification strengths. Reasonable agreement between the registration approaches and histology is found. Manual registration validates the automatic approach on the macroscopic level (top row) in terms of tissue layer boundaries and bigger blood vessels. On the mesoscopic level (middle row) the difference between the registration methods becomes more pronounced, thus highlighting the differences between smaller vessels and groups of cells. On the microscopic level (bottom) one may clearly appreciate that automatic registration does not account for feature correspondence down to the true micrometre level, as cell correspondence is not preserved. To preserve the microscopic level of correspondence between tomography and histology, further quantitative comparison was based on the results of the expert-based registration. 

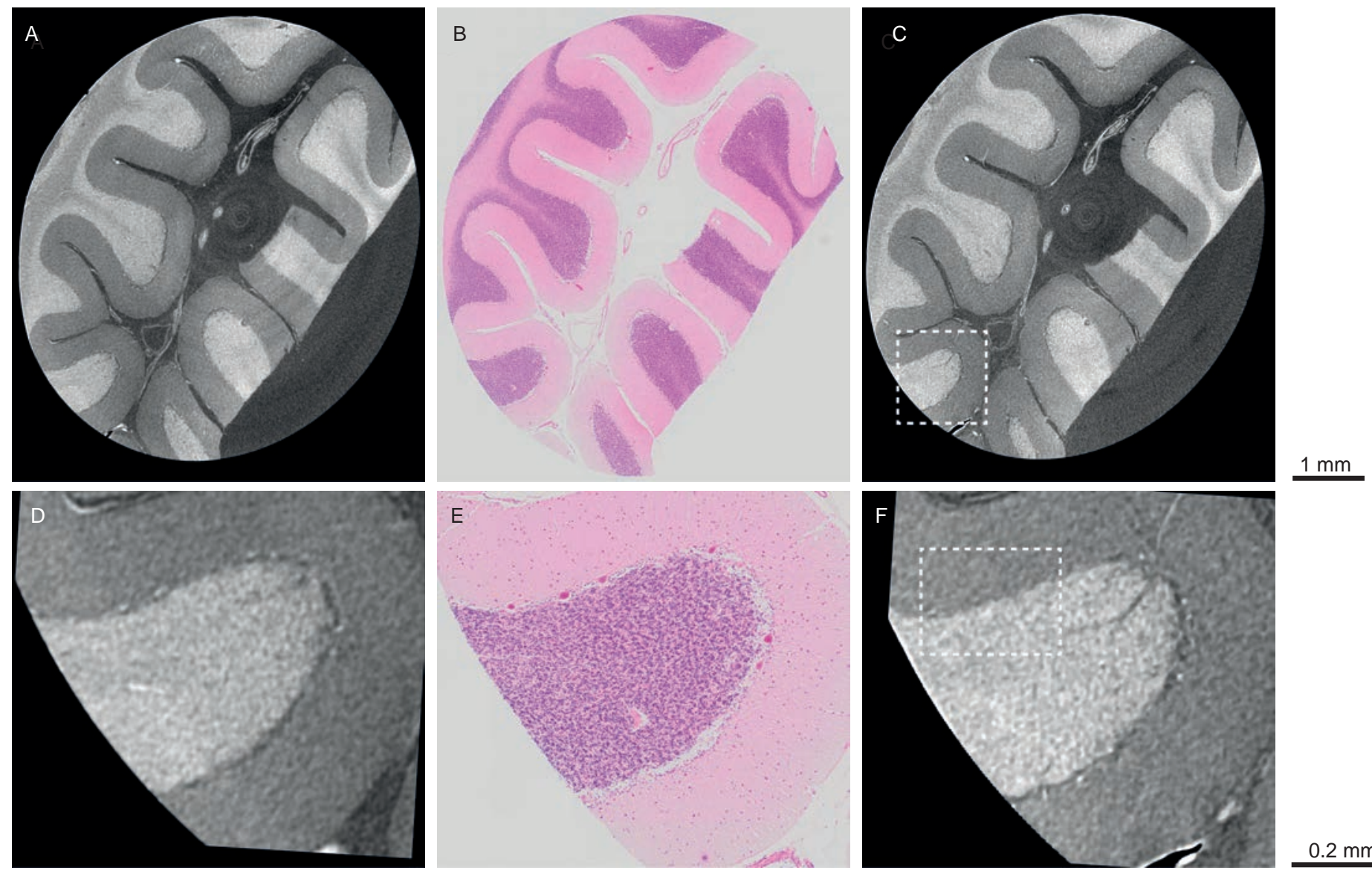

$0.2 \mathrm{~mm}$
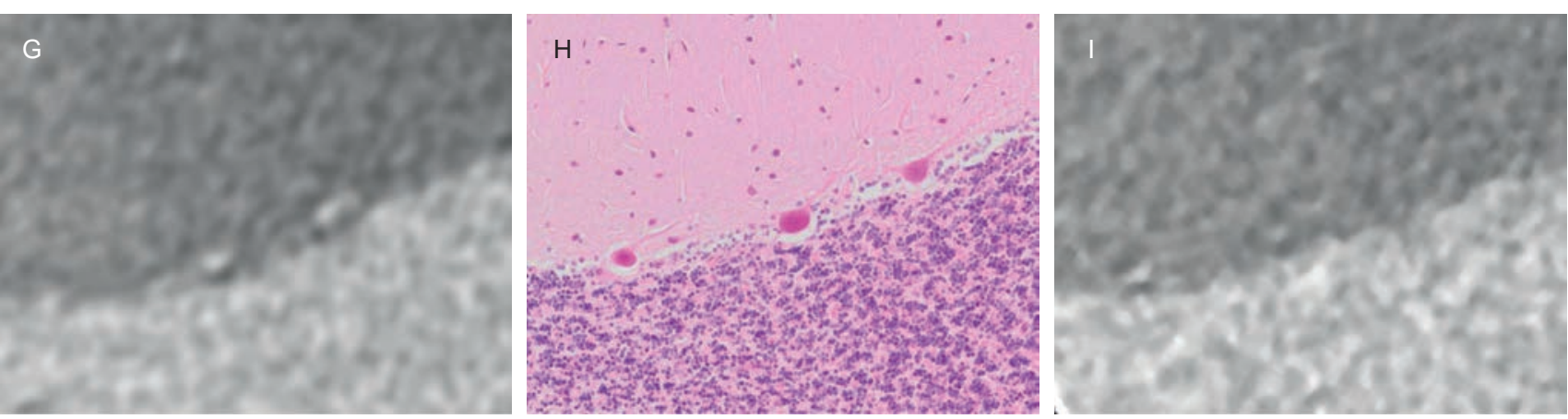

Fig. 6: Validation of tomography results by histology. The comparison between expert-based and automatic registration at three magnification levels highlights similarities in the results. By means of 2D-3D expert-based (A, D, G) and automatic $(\mathrm{C}, \mathrm{F}, \mathrm{I})$ registration, histological section (B) was positioned in the 3D tomographic volume. E, H: magnified parts of the histological section B. Tomography images are scaled from black to white in arbitrary units of X-ray attenuation.

Fig. 7 shows the histograms (left and top) of the single expert-based registered histological section and tomographic slice (right) along with a related 2D-2D joint histogram. In both modalities white matter $\left(1^{*}\right.$, green) and Stratum moleculare (1, green) appear darker than Stratum granulosum (3, pink). The white 
matter exhibits very similar grey values to the Stratum moleculare (Schulz et al., 2012) and is presented by a single histogram peak (green) for both modalities. Stratum granulosum gives rise to a separate histogram peak (pink). Blood vessels exhibit intermediate grey values between Stratum granulosum and Stratum moleculare and do not have a separate peak. A grey colour overlap between Stratum moleculare and blood vessels caused partial segmentation of blood vessels during segmentation of Stratum granulosum, as highlighted in Fig.4. Inhomogeneities in illumination during the acquisition of histology data resulted in separating the background peak into two (dark and light blue). The joint histogram shows three main data clouds associated with background (paraffin for $\mu \mathrm{CT}$ ), white matter and Stratum moleculare, as well as Stratum granulosum. The standard deviation of related histogram peaks is plotted as a red line on the joint histogram.

The joint histogram shows a correlation between the grey-scaled intensities of tomography and histology. Based on the 2D joint histogram there is a quadratic correlation $\left(y=p 1 \cdot x^{2}-p 2 \cdot x+p 3\right.$, where coefficients (with $95 \%$ confidence bounds) are p1 $=0.01(-0.01,0.03), \mathrm{p} 2=-0.12(-3.26,3.02)$ and $\mathrm{p} 3=14.69$ $(-78.52,107.90))$ between staining intensity of histology $x$ and the local X-ray attenuation of tomography y. Goodness of fit (R-square $=0.9988)$ is reasonable. Attempts to find a linear relationship between the intensities result in a decrease in the goodness of fit to 0.9734 . Fit coefficients (with $95 \%$ confidence bounds): $\mathrm{p} 2=-41.82(-20.81,62.86)$ and $\mathrm{p} 3=50.61(31.4,68.81)$. 


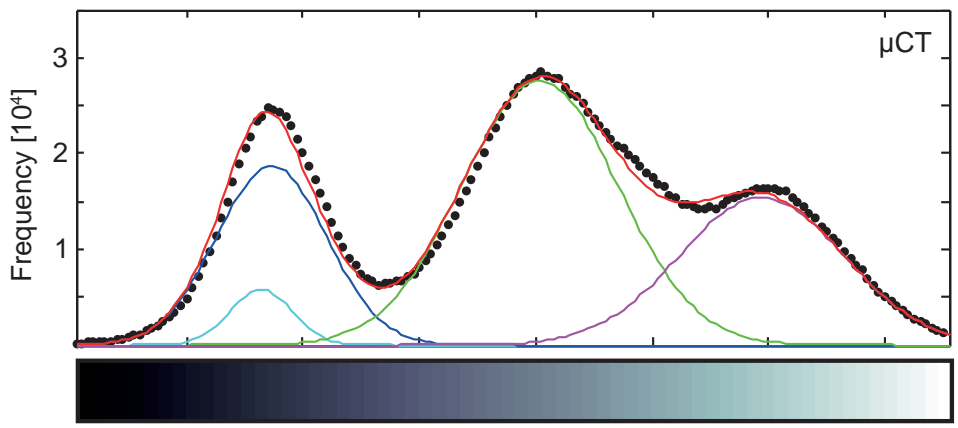

Attenuation [a.u.]
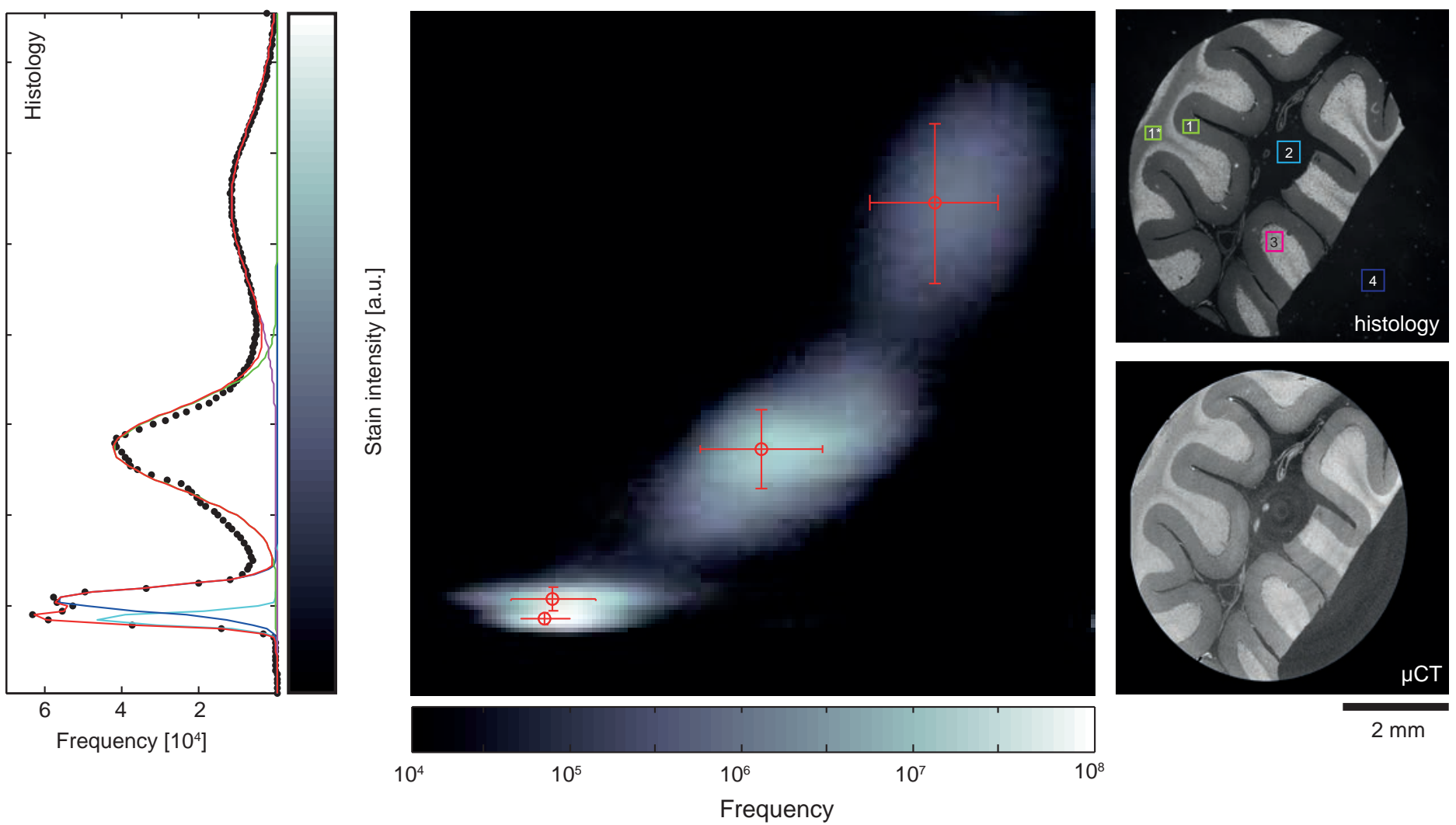

Fig. 7: Joint histogram of registered the tomographic slice and the histological section with histograms (black dots), with fitted multi-Gaussian distributions (red curves) and individual Gaussian peaks (light blue, dark blue, green and pink). Light and dark blue (2 and 4): background (paraffin for $\mu \mathrm{CT}$ ), green $\left(1\right.$ and $\left.1^{*}\right)$ : white matter and Stratum moleculare, pink (3): Stratum granulosum.

Fig. 8 shows the selected tomographic slice (A) converted into an RGB colour space of histology data (B, D). The grey-scaled tomographic slice has been transformed into a colour space based on quadratic and 245 linear interpolation, respectively, through component-wise histogram equalisation. Differences between the RGB-coloured tomography slices B and D are hardly recognisable. 

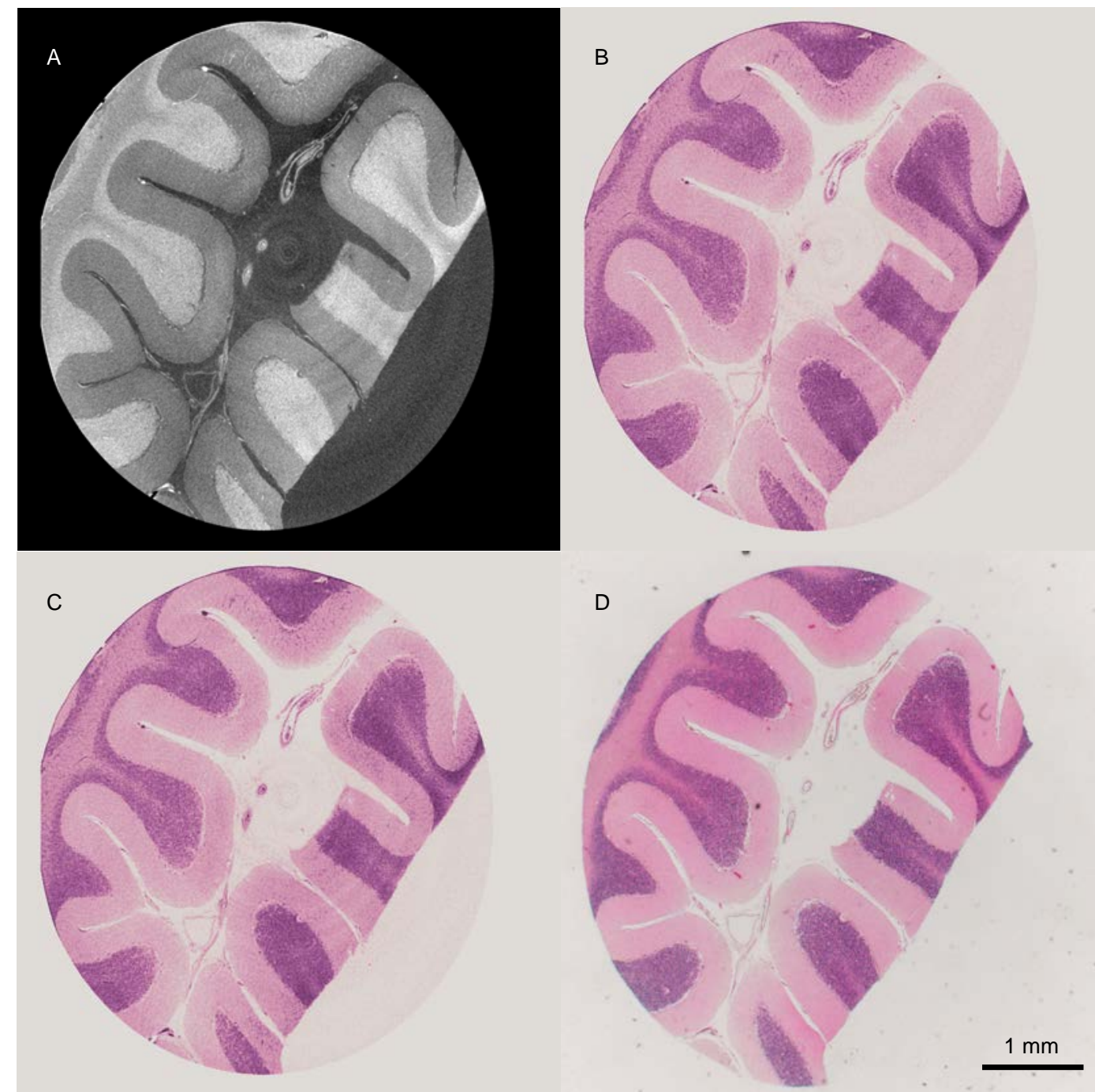

Fig. 8: Virtual histology by colourised $\mu \mathrm{CT}$ : Comparison of stain intensity and X-ray attenuation. The selected registered tomography slice (A) has been converted into an RGB colour space to resemble the H\&E-stained histological section (D). B: tomography slice coloured as histology based on the quadratic relation, C: tomography slice coloured as histology based on the linear relation.

To compare the quality of the images obtained with $\mu \mathrm{CT}$ and histology we considered the contrast-tonoise ratio between the anatomical structures given by Equation 1 , where $I_{1}, I_{2}, \sigma_{1}$ and $\sigma_{2}$ were extracted from histogram fitting. The results are shown in Table 2, Note that the increased $C N R$ of $\mu C T$ compared to Table 1 is caused by data filtering.

In general, histological data exhibit a higher contrast-to-noise ratio than tomography, as highlighted in Table 2. The highest contrast-to-noise ratio was determined to be between the background and Stratum 


\section{Table 2}

Results for the quantitative comparison of $C N R$ between the tomographic slice and the histological section (see Fig. 7). CNR (I): contrast-to-noise ratio between the background and Stratum moleculare/white matter, CNR (II): contrast-to-noise ratio between the background and Stratum granulosum, CNR (III): contrast-to-noise ratio between Stratum moleculare/white matter and Stratum granulosum, VR: volume ratio Stratum moleculare/Stratum granulosum for 2D slices (*) and 3D data.

\begin{tabular}{lccccc}
\hline & $\boldsymbol{C N R}(I)$ & $\boldsymbol{C N R}(I I)$ & $\boldsymbol{C N R}(I I I)$ & $\mathbf{V R}(*)$ & VR \\
\hline Histology & $3.65 \pm 0.10$ & $4.91 \pm 0.05$ & $2.75 \pm 0.03$ & $1.95 \pm 0.03$ & \\
$\mu \mathrm{CT}$ & $2.81 \pm 0.08$ & $4.96 \pm 0.17$ & $1.96 \pm 0.05$ & $1.93 \pm 0.05$ & $1.97 \pm 2.03 \times 10^{-4}$ \\
\hline
\end{tabular}

granulosum for both modalities. CNRs between morphological structures, calculated for the histological slices acquired with the optical magnifications $1.25 \mathrm{x}$ and $2 \mathrm{x}$ provide comparable results, with a difference less than $10 \%$ except for $C N R(I I)$, as due to resolution decrease Stratum granulosum appears less homogeneous.

\section{Discussion}

The results shown in Table 1 demonstrate that scans performed with lower voltages and tungsten targets generally exhibit a better contrast-to-noise ratio for anatomical features. This result is expected, as the selected voltages are higher than optimal for the soft tissue sample (Schulz et al., 2010b). In order to increase contrast, energy reduction is required (Grodzins, 1983a.b). Due to the insufficient counts for the low voltages in the nanotom ${ }^{\circledR} \mathrm{m}$, a further decrease in voltage is not beneficial. Therefore, the lowest possible voltage with sufficient photon counts provides the best results.

The currently available $\mu \mathrm{CT}$ systems provide isotropic micrometer resolution. It is well known, that formalin-fixed human brain tissues show limited X-ray absorption contrast. It was shown by Schulz et al. (Schulz et al. 2010b) that only marginal absorption contrast of a formalin-fixed human cerebellum using $\mathrm{SR \mu CT}$ can be achieved. Obviously, the contrast found for FFPE brain is superior (see Figs. 4 and 6). In the specific case of the cerebellum, the 3D visualization of the Purkinje cells without staining could be achieved. Dehydration of the tissue induces a structure-dependent density increase. It is speculated that dehydration also causes an increase in attenuation differences, which leads to the observed gain in contrast. The subsequently performed paraffin embedding may accentuate the attenuation differences.

A decrease of pixel size below $3.5 \mu \mathrm{m}$ enables sub-cellular resolution. The $\mu \mathrm{CT}$ system used in the present study can record data with pixel sizes down to $200 \mathrm{~nm}$ (General Electric, Measurement and Control, 
2014). This nanometer range would be sufficient to visualize the neuronal dendrite structures or vascular structure including small capillaries without contrast agent in 3D Such pixel sizes, however, restrict the sample diameters to the micrometer range. Nevertheless, the spatial resolution, which is limited by the focal spot size of around $900 \mathrm{~nm}$, for acceptable acquisition times is hardly better than one micrometer. If the user agrees with micrometer resolution, volumes of centimeter sizes can be evaluated. The multi|scan function (General Electric, Measurement and Control, 2014) enables to scan one height step after the other. In this direction, the sample size is only restricted by the size of interior of the instrument and the hub of the manipulator. Based on the specifications, the system is applicable to a wide sample range between $0.25 \mathrm{~mm}$ and $25 \mathrm{~mm}$ sample height. Thus, we conclude that $\mu \mathrm{CT}$ allows sample visualisation with cellular resolution with minor sample size limitations.

Within approximately four hours $600 \mu \mathrm{CT}$-slices have been acquired. The acquisition of a comparable number of histological sections by means of serial sectioning might need between five and ten hours. Staining with haematoxylin and eosin (H\&E) requires additional time, approximately up to 15 minutes pro slide. In addition, serial sectioning is technically challenging and individual sections can be lost. The final volume reconstruction by registration of thin sections is a complex, technically demanding process (Krauth et al., 2010) .

The comparison of histograms, as seen in Fig. 7, enables us to conclude that modalities are comparable and present very similar information for the same anatomical structures. The results suggest that $\mu \mathrm{CT}$ could become a significant complement to histological methods, as it offers the possibility of virtual histology (Gambichler et al. 2007) of physically soft tissues within a laboratory environment. Virtual cutting planes within the tomography volume can essentially simulate cuts at any angle, which would be of considerable benefit to histology, where cut direction can only partially be controlled, typically not altered and has limitations with respect to the number of sections. We specifically envision pre-sectioning scans to extrapolate histological data to 3D.

Histological examinations represent a powerful, high-resolution methodology. One has to understand that $\mathrm{H} \& \mathrm{E}$, even though it is the most common form of staining, sits well below the capabilities that histochemistry has to offer in general. Histological sections are typically stained with diverse dyes and labelling systems. Furthermore, histochemistry typically reveals biochemical data along with morphology. A qualitative comparison of the histological section (Fig. 8D) and RGB-scaled tomographic slices (Fig. 8 B 
and C) highlights similarities in the data. A combination of histology and tomography could enable virtual "multi-staining", and each histological section can be stained with one protocol highlighting particular structures of interest, while $\mu \mathrm{CT}$ can extrapolate results over the entire specimen. Moreover, $\mu \mathrm{CT}$ is non-destructive, and so the same specimen can be reused for further investigations.

In histology, staining intensity often varies significantly due to storage temperature, tissue preservation, etc. (Richendrfer et al., 2009). In addition, microscopic data are influenced by illumination conditions and the colorimetric characteristics of the camera. Combining imaging methods enables inter- or intra-sample comparisons.

The fusion of histological and tomographic data can enhance the significant benefits of multimodal imaging (Uludağ and Roebroeck, 2014) when studying tissue microstructures. Tomography can be used to complement histology techniques in clinical applications with the potential to automate quantification tasks, for example volume measurements of the entire cerebellum or its layers (Hashimoto et al., 2001; Rosin et al. 2015) or the localisation of pathological changes in FFPE specimens that could then be optimally oriented for sectioning. Measuring the volume of the cerebellum can be performed in vivo by MRI with acceptable resolutions, but the strength of $\mu \mathrm{CT}$ is clearly its micrometer range resolution, and $\mu \mathrm{CT}$ can allow for statistically significant isotropic volume or surface measurements to be performed on the entire tissue sample of microstructures such as individual cells.

Nevertheless, the merits of true isotropic visualisation are not limited to providing better volumetric measurements. Another potential application of $\mu \mathrm{CT}$ data is the correction of local distortions in histological data due to the preparation procedures (Mega et al., 1997) by applying 2D-3D registration, similar to (Schulz et al. 2010a).

\section{Conclusion}

We have demonstrated that paraffin-embedded human tissue shows sufficient absorption contrast for the discrimination of the specimen's characteristic morphology, including individual cells. Structures with diameters on the micrometer scale are visible not only in bony tissue, but also in soft materials with laboratory $\mu \mathrm{CT}$ system. Due to the observed effect, one can conclude that paraffin embedding increases the density resolution in brain tissue. Thus, laboratory-based $\mu \mathrm{CT}$ of FFPE samples can be understood as ease of use, fast and reliable tissue visualisation methodology suitable for imaging of biological specimens 
with volume in cm-range.

Furthermore, we have shown that 2D-3D registration can generate accurate correspondence and has the potential to implement 3D tomographic data into histological examinations. Moreover, 3D data can be correlated to and extend 2D histological data.

\section{Acknowledgements}

The financial support of Swiss National Science Foundation (SNSF) projects 147172, 150164 and SNSF R'Equip project 133802 is highly acknowledged. The authors are thankful to members of the BMC team for their helpful discussions, and Peter Thalmann who greatly assisted the work.

\section{References}

Ashton, J., West, J., Badea, C., 2015. In vivo small animal micro-CT using nanoparticle contrast agents. Front. Pharmacol. 6. doi:10.3389/fphar.2015.00256.

Bay, H., Ess, A., Tuytelaars, T., Van Gool, L., 2008. Speeded-Up Robust Features (SURF). Comput. Vis. Image Underst. 110, 346-359. doi:10.1016/j.cviu.2007.09.014

Beckmann, F., Herzen, J., Haibel, A., Müller, B., Schreyer, A., 2008. High density resolution in synchrotronradiation-based attenuation-contrast microtomography. Proc. SPIE 7078, 70781D. doi:10.1117/12. 794617.

Blouin, S., Moreau, M., Baslé, M., Chappard, D., 2006. Relations between radiograph texture analysis and microcomputed tomography in two rat models of bone metastases. Cells Tissues Organs 182, 182-192. doi:10.1159/000093967.

Chappard, D., Blouin, S., Libouban, H., Baslè, M.F., Audran, M., 2005. Microcomputed Tomography for the Study of Hard Tissues and Bone Biomaterials. Microsc. Anal. 19, 19-21.

Chicherova, N., Fundana, K., Müller, B., Cattin, P., 2014. Histology to $\mu$ CT Data Matching Using Landmarks and a Density Biased RANSAC. Lect. Notes Comput. Sc. (including subseries Lect. Notes Artif. Int. and Lect. N. Bioinformati.) 8673 LNCS, 243-250. doi:10.1007/978-3-319-10404-1_31. 
Chung, K., Wallace, J., Kim, S.Y., Kalyanasundaram, S., Andalman, A., Davidson, T., Mirzabekov, J., Zalocusky, K., Mattis, J., Denisin, A., Pak, S., Bernstein, H., Ramakrishnan, C., Grosenick, L., Gradinaru, V., Deisseroth, K., 2013. Structural and molecular interrogation of intact biological systems. Nature 497, 332-337. doi:10.1038/nature12107.

Costantini, I., Ghobril, J.P., Di Giovanna, A., Mascaro, A., Silvestri, L., Müllenbroich, M., Onofri, L., Conti, V., Vanzi, F., Sacconi, L., Guerrini, R., Markram, H., Iannello, G., Pavone, F., 2015. A versatile clearing agent for multi-modal brain imaging. Sci. Rep. 5, 1-5. doi:10.1038/srep09808.

de Crespigny, A., Bou-Reslan, H., Nishimura, M.C., Phillips, H., Carano, R.A.D., D’Arceuil, H.E., 2008. 3D micro-CT imaging of the postmortem brain. J. Neurosci. Meth. 171, 207-213. doi:10.1016/j . jneumeth.2008.03.006.

Dodt, H.U., Leischner, U., Schierloh, A., Jährling, N., Mauch, C., Deininger, K., Deussing, J., Eder, M., Zieglgänsberger, W., Becker, K., 2007. Ultramicroscopy: Three-dimensional visualization of neuronal networks in the whole mouse brain. Nat. Methods. 4, 331-336. doi:10.1038/nmeth1036.

Egbert, A., Brunke, O., 2011. High-resolution X-ray computed tomography for materials research. Adv. Mat. Res. 222, 48-51. doi:10.4028/www.scientific.net/AMR .222.48.

Feldkamp, I., Davis, L., Kress, J., 1984. Practical cone-beam algorithm. J. Opt. Soc. Am. A Opt. Image Sci. Vis. 1, 612-619.

Fischler, M., Bolles, R., 1981. Random sample consensus: a paradigm for model fitting with applications to image analysis and automated cartography. Commun. ACM 24, 381-395. doi:10.1145/358669. 358692 .

Fuchs, T., Buhmann, J., 2011. Computational pathology: Challenges and promises for tissue analysis Comput. Med. Imag. Grap. 35, 515-530. doi:10.1016/j.compmedimag.2011.02.006.

Gambichler, T., Moussa, G., Regeniter, P., Kasseck, C., Hofmann, M., Bechara, F., Sand, M., Altmeyer, P., Hoffmann, K., 2007. Validation of optical coherence tomography in vivo using cryostat histology. Phys. Med. Biol. 52, N75-N85. doi:10.1088/0031-9155/52/5/N01. 
General Electric, Measurement and Control, 2014. phoenix nanotom m $180 \mathrm{kv} / 20 \mathrm{w}$ X-ray nanoCT system for high-resolution analysis and 3D metrology. URL: https://www.gemeasurement.com/ inspection-ndt/radiography-and-computed-tomography/phoenix-nanotom-m.

Germann, M., Morel, A., Beckmann, F., Andronache, A., Jeanmonod, D., Müller, B., 2008. Strain fields in histological slices of brain tissue determined by synchrotron radiation-based micro computed tomography, J. Neurosci. Meth. 170, 149-155. doi:10.1016/j.jneumeth.2008.01.011.

Grodzins, L., 1983a. Optimum energies for x-ray transmission tomography of small samples. Applications of synchrotron radiation to computerized tomography I. Nucl. Instr. Meth. Phys. Res. 206, 541-545. doi:10.1016/0167-5087(83)90393-9.

Grodzins, L., 1983b. Critical absorption tomography of small samples. Proposed applications of synchrotron radiation to computerized tomography II. Nucl. Instr. Meth. Phys. Res. 206, 547-552. doi:10.1016/ $0167-5087$ (83)90394-0.

Guk, B., Yae, J., Tae, J., Hwa, S., Yong, S., Sang, J., 2008. X-ray imaging of various biological samples using a phase-contrast hard X-ray microscope. Microsc. Res. Techniq. 71, 639-643. doi:10.1002/ jemt.20601.

Hashimoto, H., Shintani, N., Tanaka, K., Mori, W., Hirose, M., Matsuda, T., Sakaue, M., Miyazaki, J.I., Niwa, H., Tashiro, F., Yamamoto, K., Koga, K., Tomimoto, S., Kunugi, A., Suetake, S., Baba, A., 2001. Altered psychomotor behaviors in mice lacking pituitary adenylate cyclase-activating polypeptide (PACAP). P. Natl. Acad. Sci. USA 98, 13355-13360. doi:10.1073/pnas.231094498.

Huang, S., Kou, B., Chi, Y., Xi, Y., Cao, Y., Cui, W., Hu, X., Shao, Z., Guo, H., Fu, Y., Xiao, T., Sun, J., Zhao, J., Wang, Y., Wu, J., 2015. In-line phase-contrast and grating-based phase-contrast synchrotron imaging study of brain micrometastasis of breast cancer. Sci. Rep. 5. doi:10.1038/srep09418.

Irshad, H., Veillard, A., Roux, L., Racoceanu, D., 2014. Methods for nuclei detection, segmentation, and classification in digital histopathology: A review-current status and future potential. IEEE Rev. Biomed. Eng. 7, 97-114. doi:10.1109/RBME.2013.2295804. 
Kandel, E.R., Schwartz, J.H., Jessell, T.M., Siegelbaum, S.A., Hudspeth, A.J., 2012. Principles of Neural Science (Kandel). McGraw-Hill Education/Medical; 5th edition.

Krauth, A., Blanc, R., Poveda, A., Jeanmonod, D., Morel, A., Székely, G., 2010. A mean three-dimensional atlas of the human thalamus: Generation from multiple histological data. NeuroImage 49, 2053-2062. doi:10.1016/j.neuroimage.2009.10.042.

Kroon, D.J., Slump, C., 2009. MRI modalitiy transformation in demon registration. Proc. ISBI: From Nano to Macro 1, 963-966. doi:10.1109/ISBI.2009.5193214.

Lang, S., Zanette, I., Dominietto, M., Langer, M., Rack, A., Schulz, G., Le Duc, G., David, C., Mohr, J., Pfeiffer, F., Müller, B., Weitkamp, T., 2014. Experimental comparison of grating- and propagationbased hard X-ray phase tomography of soft tissue. J. Appl. Phys. 116, 116. doi:10.1063/1.4897225.

Langer, M., Pacureanu, A., Suhonen, H., Grimal, Q., Cloetens, P., Peyrin, F., 2012. X-Ray Phase Nanotomography Resolves the 3D Human Bone Ultrastructurey, PLoS ONE 7. doi:10.1371/journal. pone.0035691

Lareida, A., Beckmann, F., Schrott-Fischer, A., Glueckert, R., Freysinger, W., Müller, B., 2009. Highresolution X-ray tomography of the human inner ear: synchrotron radiation-based study of nerve fibre bundles, membranes and ganglion cells. J. Microsc. 234, 95-102. doi:10.1111/j.1365-2818.2009. $03143 . x$.

Manjon-Herrera, J.V., 2006. EM image segmentation. URL: http://www.mathworks.com/ matlabcentral/fileexchange/10956-em-image-segmentation

Markelj, P., Tomaževič, D., Likar, B., Pernuš, F., 2012. A review of 3D/2D registration methods for image-guided interventions. Med. Image Anal. 16, 642-661. doi:10.1016/j.media.2010.03.005.

Mega, M.S., Chen, S.S., Thompson, P.M., Woods, R.P., Karaca, T.J., Tiwari, A., Vinters, H.V., Small, G.W., Toga, A.W., 1997. Mapping histology to metabolism: Coregistration of stained whole-brain sections to premortem PET in Alzheimer's disease. NeuroImage 5, 147-153. doi:10.1006/nimg.1996. 0255 . 
Metscher, B.D., 2009. MicroCT for comparative morphology: simple staining methods allow highcontrast 3D imaging of diverse non-mineralized animal tissues. BMC Physiol. 9, 1-14. doi:10.1186/ 1472-6793-9-11.

Mokso, R., Cloetens, P., Maire, E., Ludwig, W., Buffìre, J.Y., 2007. Nanoscale zoom tomography with hard x rays using Kirkpatrick-Baez optics. Appl. Phys. Lett. 90. doi:10.1063/1.2719653.

Müller, B., Beckmann, F., Huser, M., Maspero, F., Szekely, G., Ruffieux, K., Thurner, P., Wintermantel, E., 2002. Non-destructive three-dimensional evaluation of a polymer sponge by micro-tomography using synchrotron radiationy. Biomol. Eng. 19, 73-8. doi:10.1016/S1389-0344(02)00014-X.

Müller, B., Deyhle, H., Lang, S., Schulz, G., Bormann, T., Fierz, F., Hieber, S., 2012. Three-dimensional registration of tomography data for quantification in biomaterials science. Int. J. Mater. Res. 103, 242-249. doi:10.3139/146.110663.

Müller, B., Riedel, M., Thurner, P.J., 2006. Three-dimensional characterization of cell clusters using synchrotron-radiation-based micro-computed tomography. Microsc. Microanal. 12, 97-105. doi:10. $1017 / \mathrm{S} 1431927606060168$.

Murray, E., Cho, J., Goodwin, D., Ku, T., Swaney, J., Kim, S.Y., Choi, H., Park, Y.G., Park, J.Y., Hubbert, A., McCue, M., Vassallo, S., Bakh, N., Frosch, M., Wedeen, V., Seung, H., Chung, K., 2015. Simple, Scalable Proteomic Imaging for High-Dimensional Profiling of Intact Systems. Cell 163, 1500-1514. doi:10.1016/j.cell.2015.11.025.

Ribi, W., Senden, T.J., Sakellariou, A., Limaye, A., Zhang, S., 2008. Imaging honey bee brain anatomy with micro-X-ray-computed tomography, J. Neurosci. Meth. 171, 93-97. doi:10.1016/j.jneumeth. 2008.02 .010 .

Richardson, D., Lichtman, J., 2015. Clarifying Tissue Clearing. Cell 162, 246-257. doi:10.1016/j.cel1. 2015.06 .067 .

Richendrfer, H., Wetzel, J., Swann, J., 2009. Temperature, peroxide concentration, and immunohistochemical staining method affects staining intensity, distribution, and background. Appl. Immunohistochem. Mol. Morphol. 17, 543-546. doi:10.1097/PAI.0b013e3181a91595. 
Rosin, J., McAllister, B., Dyck, R., Percival, C., Kurrasch, D., Cobb, J., 2015. Mice lacking the transcription factor SHOX2 display impaired cerebellar development and deficits in motor coordination. Dev. Biol. 399, 54-67. doi:10.1016/j.ydbio.2014.12.013.

Schulz, G., Morel, A., Imholz, M., Deyhle, H., Weitkamp, T., Zanette, I., Pfeiffer, F., David, C., MüllerGerbl, M., Müller, B., 2010a. Evaluating the microstructure of human brain tissues using synchrotron radiation-based micro computed tomography, Proc. SPIE 7804, 78040F. doi:10.1117/12.859273.

Schulz, G., Waschkies, C., Pfeiffer, F., Zanette, I., Weitkamp, T., David, C., Müller, B., 2012. Multimodal imaging of human cerebellum - merging X-ray phase microtomography, magnetic resonance microscopy and histology. Sci. Rep. 2, 826. doi:10.1038/srep00826.

Schulz, G., Weitkamp, T., Zanette, I., Pfeiffer, F., Beckmann, F., David, C., Rutishauser, S., Reznikova, E., Müller, B., 2010b. High-resolution tomographic imaging of a human cerebellum: comparison of absorption and grating-based phase contrast. J. R. Soc. Interface 7, 1665-1676. doi:10.1098/rsif. 2010.0281 .

Silvestri, L., Bria, A., Sacconi, L., Iannello, G., Pavone, F., 2012. Confocal light sheet microscopy: Micronscale neuroanatomy of the entire mouse brain. Opt. Express 20, 20582-20598. doi:10.1364/0E.20. 020582 .

So, P., 2002. Encyclopedia of life sciences, Two-photon fluorescence light microscopy. Macmillan Publishers Ltd, Nature Publishing Group.

Sombke, A., Lipke, E., Michalik, P., Uhl, G., Harzsch, S., 2015. Potential and limitations of X-Ray micro-computed tomography in arthropod neuroanatomy: A methodological and comparative survey. Journal of Comparative Neurology 523, 1281-1295. doi:10.1002/cne.23741.

Stalder, A., Ilgenstein, B., Chicherova, N., Deyhle, H., Beckmann, F., Müller, B., Hieber, S., 2014. Combined use of micro computed tomography and histology to evaluate the regenerative capacity of bone grafting materials. Int. J. Mat. Res. 105, 679-691. doi:10.3139/146.111050.

Thimm, B., Hofmann, S., Schneider, P., Carretta, R., Müller, R., 2012. Imaging of cellular spread on a 
three-dimensional scaffold by means of a novel cell-labeling technique for high-resolution computed tomography. Tissue Eng. - Part C: Methods 18, 167-175. doi:10.1089/ten.tec.2011.0262.

Uludağ, K., Roebroeck, A., 2014. General overview on the merits of multimodal neuroimaging data fusion. NeuroImage 102, 3-10. doi:10.1016/j.neuroimage.2014.05.018.

Vladimirov, N., Mu, Y., Kawashima, T., Bennett, D., Yang, C.T., Looger, L., Keller, P., Freeman, J., Ahrens, M., 2014. Light-sheet functional imaging in fictively behaving zebrafish. Nat. Methods 11, 883-884. doi:10.1038/nmeth.3040

„Wang, H., Zhu, J., Akkin, T., 2014. Serial optical coherence scanner for large-scale brain imaging at microscopic resolution. NeuroImage 84, 1007-1017. doi:10.1016/j.neuroimage.2013.09.063.

Wenz, J., Schleede, S., Khrennikov, K., Bech, M., Thibault, P., Heigoldt, M., Pfeiffer, F., Karsch, S., 2015. Quantitative X-ray phase-contrast microtomography from a compact laser-driven betatron source. Nat. Commun. 6. doi:10.1038/ncomms 8568 .

Wolf, S., Supatto, W., Debrégeas, G., Mahou, P., Kruglik, S., Sintes, J.M., Beaurepaire, E., Candelier, R., 2015. Whole-brain functional imaging with two-photon light-sheet microscopy, Nat. Methods 12, 379-380. doi:10.1038/nmeth.3371.

Zehbe, R., Haibel, A., Riesemeier, H., Gross, U., Kirkpatrick, C., Schubert, H., Brochhausen, C., 2010. Going beyond histology. Synchrotron micro-computed tomography as a methodology for biological tissue characterization: From tissue morphology to individual cells. J. R. Soc. Interface 7, 49-59. doi:10.1098/rsif.2008.0539.

Zehbe, R., Schmitt, V.H., Kirkpatrick, C.J., Brochhausen, C., 2015. High resolution X-ray tomography Three-dimensional characterisation of cell-scaffold constructs for cartilage tissue engineering. Mater. Sci. Tech. (UK) 31, 167-173. doi:10.1179/1743284714Y.0000000667. 


\section{List of Figures}

1 Shape of the histological section in the tomography dataset based on the expert-based (black) and automatic (red) registrations. Note that automatic registration yields a plane, while manual registration allows for curved surfaces. Arrows indicate regions of low landmark

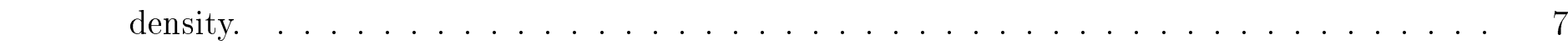

2 Visualisation of characteristic landmarks for manual 2D-3D registration marked in a selected histological section (A) and in the $\mu \mathrm{CT}$ dataset (B). Characteristic landmarks of one

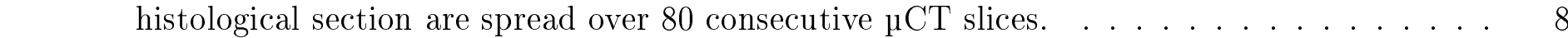

$3 \quad$ Human cerebellum block measured with six $\mu \mathrm{CT}$ settings. Images provide sufficient contrast for visualising morphological features. As the images are dominated by noise, filtering is required. A-F: selected part of the tomographic slice recorded with parameters summarised in Table 1. $\mathrm{F}^{*}$ : Slice F filtered for noise reduction. 1 (yellow): Purkinje cell, 2 (pink): Stratum granulosum, 3 (light green): Stratum moleculare, 4 (dark green): blood vessel within the white matter. . . . . . . . . . . . . . . . . . . . 9

$4 \quad$ The 3D-rendering of the human cerebellum block based on the filtered $\mu \mathrm{CT}$ data (A) shows blood vessels of various sizes, Stratum moleculare, Stratum granulosum, and white matter. Intensity thresholding (B) enables the exclusion of Stratum moleculare for an improved visualisation of the blood vessels and Purkinje cells $(\mathrm{C}, \mathrm{D})$. . . . . . . . . . .

$5 \quad$ Virtual cutting planes within the $\mu \mathrm{CT}$ volume visualising the position of the histological sections. The colour on top of the registered $\mu \mathrm{CT}$ slices $(\mathrm{E}-\mathrm{H})$ indicates the shape of the

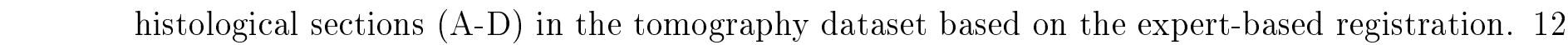

$6 \quad$ Validation of tomography results by histology. The comparison between expert-based and automatic registration at three magnification levels highlights similarities in the results. By means of 2D-3D expert-based (A, D, G) and automatic (C, F, I) registration, histological section (B) was positioned in the 3D tomographic volume. E, H: magnified parts of the histological section B. Tomography images are scaled from black to white in arbitrary units of X-ray attenuation. . . . . . . . . . . . . . . . . . . . 
7 Joint histogram of registered the tomographic slice and the histological section with histograms (black dots), with fitted multi-Gaussian distributions (red curves) and individual Gaussian peaks (light blue, dark blue, green and pink). Light and dark blue (2 and 4): background (paraffin for $\mu \mathrm{CT}$ ), green $\left(1\right.$ and $\left.1^{*}\right)$ : white matter and Stratum moleculare, pink (3): Stratum granulosum. . . . . . . . . . . . . . . . . 16

$8 \quad$ Virtual histology by colourised $\mu \mathrm{CT}$ : Comparison of stain intensity and X-ray attenuation. The selected registered tomography slice $(\mathrm{A})$ has been converted into an RGB colour space to resemble the H\&E-stained histological section (D). B: tomography slice coloured as histology based on the quadratic relation, C: tomography slice coloured as histology based on the linear

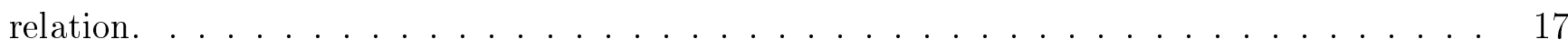

$9 \quad$ Virtual histology by colour-coded $\mu \mathrm{CT}$. Extension of 2D histological data into a third dimension by laboratory-based micro tomography. 1: white matter, 2: Stratum moleculare, 3: Stratum granulosum, 4: Purkinje cell. . . . . . . . . . . . . . . . . .

\section{List of Tables}

$1 \quad$ Selection of the optimized settings: Scanning parameters of the nanotom ${ }^{\circledR} \mathrm{m}$ used for the $\mu \mathrm{CT}$ experiments and contrast-to-noise ratios (CNRs) of the reconstructed data. Target: X-ray tube transmission target, $U$ : acceleration voltage, $I$ : e-beam current, FDD: focusdetector distance, FOD: focus-object distance, $t$ : exposure time per projection, $\mathrm{CNR}(I)$ : contrast-to-noise ratio between paraffin and Stratum moleculare; CNR $(I I)$ : contrast-tonoise ratio between paraffin and white matter; CNR $(I I I)$ : contrast-to-noise ratio between paraffin and Stratum granulosum. . . . . . . . . . . . . . . . . . . . . 10

2 Results for the quantitative comparison of $C N R$ between the tomographic slice and the histological section (see Fig. 7). $C N R(I)$ : contrast-to-noise ratio between the background and Stratum moleculare/white matter, $C N R(I I)$ : contrast-to-noise ratio between the background and Stratum granulosum, CNR (III): contrast-to-noise ratio between Stratum moleculare/white matter and Stratum granulosum, VR: volume ratio Stratum moleculare/Stratum granulosum for $2 \mathrm{D}$ slices $(*)$ and $3 \mathrm{D}$ data $\ldots \ldots \ldots \ldots \ldots$. . . . . . . . . 


\section{Graphical ${ }^{\top} \bar{\top}$ bstract}

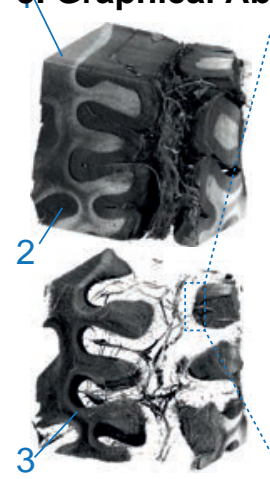

Histology

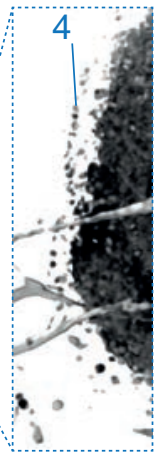

$+$

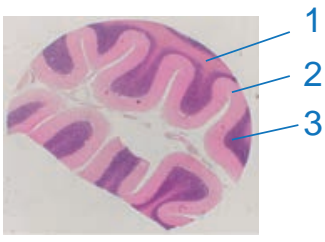

1

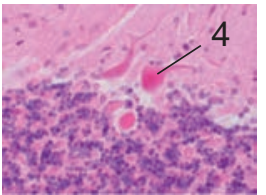

Three-dimensional microanatomy

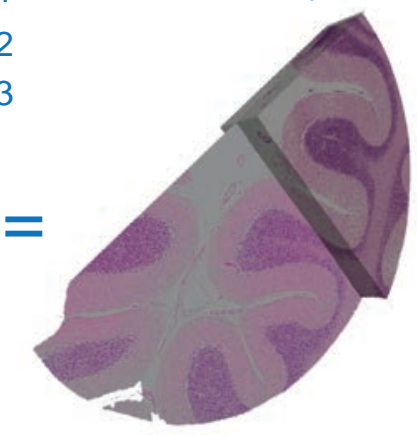

